\title{
Exploration du rôle potentiel des pharmacies privées dans l'offre de services de planification familiale au Sénégal: analyse secondaire es données de ADEMAS
}

\author{
Fatou Mbow \\ Population Council \\ Amadou Korka Diallo \\ Nafissatou Diop \\ Population Council \\ Sérigne Momar Kane \\ Cheikh Saad Bou Sarr
}

See next page for additional authors

Follow this and additional works at: https://knowledgecommons.popcouncil.org/departments_sbsr-rh How does access to this work benefit you? Let us know!

\section{Recommended Citation}

Mbow, Fatou, Amadou Korka Diallo, Nafissatou Diop, Sérigne Momar Kane, Cheikh Saad Bou Sarr, and Sara Chace Dwyer. 2018. "Exploration du rôle potentiel des pharmacies privées dans l'offre de services de planification familiale au Sénégal: analyse secondaire es données de ADEMAS." Dakar: Population Council, The Evidence Project. 


\section{Authors}

Fatou Mbow, Amadou Korka Diallo, Nafissatou Diop, Sérigne Momar Kane, Cheikh Saad Bou Sarr, and Sara Chace Dwyer 
:

\section{Exploration du rôle potentiel des} pharmacies privées dans l'offre de services de planification familiale au Sénégal

Fatou Bintou Mbow

Amadou Korka Diallo

Nafissatou Diop

Sérigne Momar Kane

Cheikh Saad Bou Sarr

Sara Chace Dwyer

ANALYSE SECONDAIRE DES DONNÉES DE ADEMS

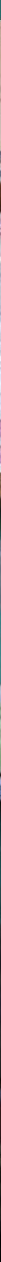





\section{Exploration du rôle potentiel des pharmacies privées dans l'offre de services de planification familiale au Sénégal}

ANALYSE SECONDAIRE DES DONNÉES DE ADEMAS

Fatou Bintou Mbow, Population Council

Amadou Korka Diallo, ADEMAS

Nafissatou Diop, Population Council

Sérigne Momar Kane, ADEMAS

Cheikh Saad Bou Sarr, ADEMAS

Sara Chace Dwyer, Population Council

MAI 2018 
Le Projet Evidence est rendu possible grâce au généreux support du Peuple Américain à travers l'Agence des Etats Unis pour le Développement International (USAID) sous les termes de l'accord de coopération no. AIDOAA-A-13-00087. Le contenu de ce document est la seule responsabilité du Projet Evidence et ne reflète pas nécessairement les points de vue de I'USAID ou du Gouvernement des Etats Unis.

\section{Evidence}

Le Projet Evidence utilise la science de la mise en œuvre -la production, traduction, et utilisation stratégique d'évidences - pour renforcer et mettre à l'échelle des programmes de planification familiale et de santé de la reproduction afin de réduire les grossesses non désirées partout dans le monde. Le Projet Evidence est dirigé par le Population Council en partenariat avec Population Reference Bureau.

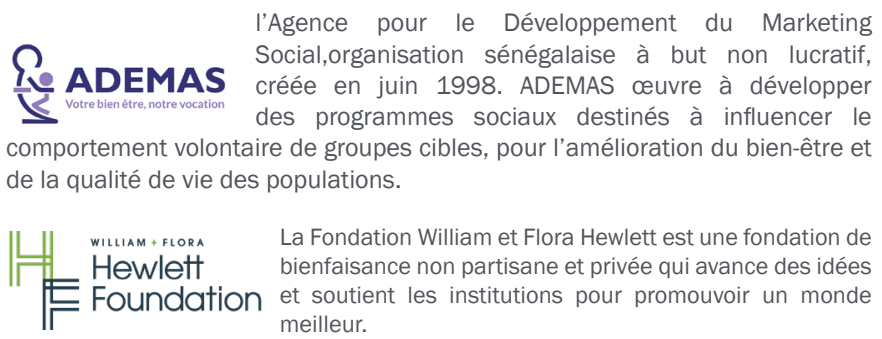

The Evidence Project

Population Council

Sacré Cœur Pyrotechnie

Appartement 2ème Etage à Droite

Dakar

Sénégal

21027 Dakar Ponty

tél: +221.33.859.5300

evidenceproject@popcouncil.org

\section{ADEMAS}

Lot $\mathrm{n}^{\circ} 112$

Sacré-coeur Pyrotechnie

BP 17603

Dakar-Liberté

Sénégal

tél: +221.33.865.01.88

ademas.sn

Publié en Mai 2018.

Citation recommandée: Mbow, Fatou Bintou, Amadou Korka Diallo, Nafissatou Diop, Sérigne Momar Kane, Cheikh Saad Bou Sarr, et Sara Chace Dwyer. 2018. "Exploration du rôle potentiel des pharmacies privées dans l'offre de services de planification familiale au Sénégal”, Analyse secondaire des données de ADEMAS. Dakar, Sénégal: Population Council, Le Projet Evidence.

(C) 2018 Le Population Council, Inc. 


\section{Table des matières}

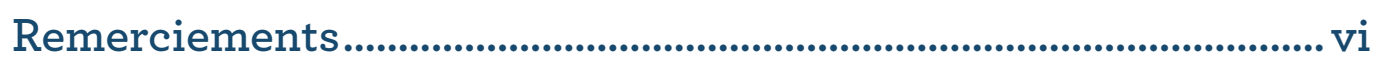

Acronymes et abréviations .................................................................... vii

Résumé exécutif ........................................................................... 1

Executive summary (English) .................................................................. 3

Introduction ......................................................................................................... 5

Contexte et justification ................................................................ 6

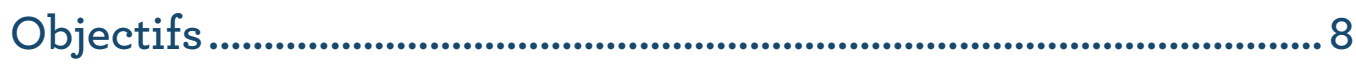

Méthodologie ................................................................................................... 9

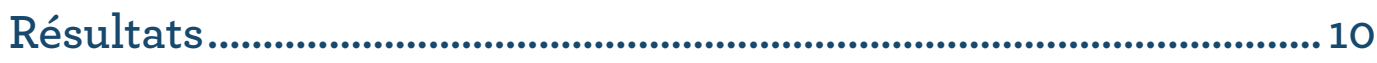

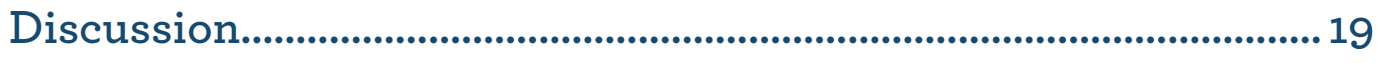

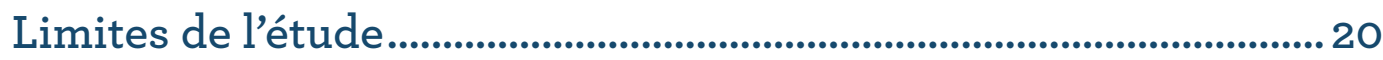

Recommandations ..................................................................................... 21

Conclusion ............................................................................................................ 22

Références bibliographiques ........................................................ 23v 


\section{Remerciements}

Nous remercions l'ADEMAS qui a contribué à la réalisation de cette analyse secondaire en mettant à notre disposition la documentation utile. Mais également pour avoir fourni et exploité les bases de données.

Cette recherche a été réalisée grâce au soutien financier de l'Agence des États-Unis pour le Développement International (USAID) à travers le Projet Evidence, et l'appui de la Fondation William et Flora Hewlett à travers un financement au Population Council. 


\title{
Acronymes et abréviations
}

\author{
ADEMAS Agence pour le Développement du Marketing Social \\ Co Contraceptifs oraux \\ CU Contraception d'urgence \\ DIU Dispositif intra-utérin \\ DSRSE Direction de la Santé de la Reproduction et de la Survie de l'Enfant \\ EDS-C Enquête Démographique et de Santé Continu \\ EDS-MICS Enquête Démographique et de Santé à Indicateurs Multiples \\ IST Infection sexuellement transmissible \\ MAP Mesure de l'Accès et de la Performance \\ MSAS Ministère de la Santé et de l'Action Sociale \\ PANPF Plan d'action national de Planification Familiale \\ PF Planification familiale \\ SIDA Syndrome d'immunodéficience acquise \\ TPCm Taux de prévalence contraceptive moderne \\ USAID Agence des États-Unis pour le Développement International
}





\section{Résumé exécutif}

En 2012, la Direction de la Santé de la Reproduction et de la Survie de l'Enfant (DSRSE) du Ministère de la Santé et de l'Action Sociale du Sénégal a adopté un ambitieux Plan d'Action National de Planification Familiale pour la période 2012-2015. L'objectif de ce plan qui s'appuie sur une approche multisectorielle était d'augmenter le taux de prévalence contraceptive moderne de $12 \%$ en 2012 à $27 \%$ en 2015 et à 45\% en 2020. Depuis sa mise en œuvre, le Programme Planification Familiale du pays a fait des progrès considérables grâce en partie à l'implication active du niveau communautaire et des organisations à but non lucratif dans la promotion et l'offre de services PF. Cependant, les pharmacies privées ne sont pas, pour le moment véritablement impliquées dans l'offre de PF car la législation sénégalaise limite leurs interventions en matière de PF à la vente de produits contraceptifs et à la fourniture de conseils aux utilisateurs potentiels. Ainsi en 2015, le Population Council a mené, dans le cadre du Projet Evidence, une étude exploratoire sur le rôle potentiel des pharmacies privées dans l'offre de services de PF, en collaboration avec la DSRSE, le Bureau santé de Agence des États-Unis pour le Développement International (USAID) Sénégal, I'Unité de Coordination du Partenariat de Ouagadougou, l'Agence pour le Développement du Marketing Social (ADEMAS) et l'Institut de Population, Développement et Santé de la Reproduction de I'Université Cheikh Anta Diop de Dakar. L'étude a été financée par I'USAID et par la Fondation William et Flora Hewlett. Pour obtenir une meilleure perception de l'expérience des pharmacies privées dans l'offre des services de PF, l'une des composantes de l'étude était de faire une analyse secondaire des données existantes de l'ADEMAS. Les évidences de cette étude pourront soutenir les efforts de plaidoyer en faveur d'une plus grande implication des pharmacies privées dans l'offre de services de PF, dans le cadre de l'approche multisectorielle adoptée par le Gouvernement du Sénégal afin de mieux répondre aux besoins de PF et de santé sexuelle et reproductive des personnes et des couples au Sénégal.

\section{Méthodologie}

Cette composante a consisté en une analyse secondaire des données existantes de deux (2) études sur les pharmacies privées. Les bases de données des études susmentionnées ont été fournies et exploitées par ADEMAS. II a été procédé à une analyse descriptive uni-variée et bi-variée des variables et une compréhension de la relation entre ces variables.

\section{Résultats}

Basé sur les données du Mesure de l'Accès et de la Performance (MAP) 2013 (menées auprès de 29 et 52 pharmacies privées) et MAP 2015 (menées menée auprès de 80 établissements de santé publique et 49 pharmacies privées), un plus grand pourcentage des pharmacies privées distribue des produits contraceptifs comparé à celui des formations sanitaires. En l'espace de deux ans, les pharmacies impliquées dans la distribution de produits contraceptifs a nettement progressé, passant de 88\% en 2013 à 100\% en 2015. Cette évolution diffère, toutefois, selon le produit contraceptif. Toutes les pharmacies interrogées distribuent la contraception orale (CO) sous plusieurs marques. Concernant les injectables, en 2015, il y avait peu de différence dans les niveaux de distribution des pharmacies et des formations sanitaires, avec respectivement $72 \%$ et $71 \%$. Les principales raisons de la non-vente des injectables dans certaines pharmacies privées étaient les 
suivantes : absence de demande pour cette méthode (50\%); méthode réservée au secteur public (29\%) ; produit jamais proposé par les distributeurs (14\%) ; arrêt momentané de la vente (7\%). Cependant 36\% des pharmacies seraient intéressées par la vente d'injectables. En 2015, la présence de la contraception d'urgence (CU) était plus importante au niveau des pharmacies visitées (78\%) que dans les formations sanitaires (63\%), et plusieurs marques y étaient proposées. Cependant $32 \%$ de ces pharmacies seraient intéressées par la vente de la CU. Peu de pharmacies privées ont déclaré vendre des implants et des DIU.

Concernant les capacités techniques du personnel des pharmacies à offrir des services de PF, il a été relevé ce qui suit : en 2011, moins d'un cinquième du personnel des pharmacies avait un bon niveau de connaissance (a pu faire une bonne classification par exemple, ils ont pu identifier et classer les méthodes selon leur durée d'action et leur efficacité) des méthodes contraceptives, et un peu plus de la moitié avait une connaissance plutôt moyenne (le personnel a pu citer les méthodes sans pouvoir les classer). La moitié des pharmaciens et un tiers des agents de comptoir avaient un bon niveau de connaissance sur la classification des types de pilules combinées. La moitié des pharmaciens et les 3/4 des agents de comptoir avaient un faible niveau de connaissance des effets secondaires ou des situations nécessitant une référence. Face à une demande de pilule pour une première utilisation de la PF, plus de la moitié du personnel des pharmacies ne respectait pas la législation en vigueur (ex : délivrance de pilule sans présentation d'une ordonnance venant d'un prescripteur agréé).

\section{Conclusion}

Les pharmacies privées présentent une meilleure disponibilité de certains produits PF que les formations sanitaires. Les capacités techniques du personnel des pharmacies privées à offrir les services de PF sont insuffisantes et devraient être renforcées. Une part importante de pharmacies privées offre certaines méthodes de PF malgré les restrictions juridiques. Audelà de la vente et du conseil, le personnel des pharmacies privées peut jouer un rôle plus important dans l'offre de services de PF, à condition de relever certains défis. 


\section{Executive summary (English)}

In 2012, the Directorate of Reproductive Health and Child Survival (DSRSE) of the Ministry of Health and Social Action of Senegal adopted the ambitious National Family Planning Action Plan for 2012-2015. The plan had a target of reaching a modern contraceptive prevalence rate of $12 \%$ by $2012,27 \%$ by 2015 and $45 \%$ by 2020 . Since its implementation, the country's family planning (FP) program has made considerable progress, due in part to the active involvement of community and non-profit organizations in the delivery of FP services. However, private pharmacies have not been actively involved in the provision of FP services due to laws limiting their role to selling contraceptives with a prescription and providing guidance on drug indications. In 2015, the Population Council, in collaboration with the DSRSE, the U.S. Agency for International Development (USAID) Senegal Health Office, the Agency for the Development of Social Marketing (ADEMAS), the Ouagadougou Partnership Coordination Unit, and the Institute of Population, Development and Reproductive Health, Cheikh Anta Diop University of Dakar, conducted an exploratory study on the potential role of private pharmacies in the provision of FP services in Senegal. The study was funded by USAID, through the Evidence Project, and the William and Flora Hewlett Foundation. One component of the study was a secondary data analysis of ADEMAS' data on private pharmacies. The analysis provides a better understanding of private pharmacies' experience providing family planning services. Evidence from this study will support advocacy efforts to increase private pharmacy involvement in the provision of FP services. This is in-line with the multi-sectoral approach adopted by the Government of Senegal to better respond to FP and sexual and reproductive health needs of individuals and couples in Senegal.

\section{Methodology}

This study component consisted of a secondary analysis of data from two studies on private pharmacies in Senegal. ADEMAS provided the data and collaborated on the analysis. Descriptive univariate and bivariate analyses were conducted.

\section{Results}

According to data from the Mesure de l'Accès et de la Performance (MAP) 2013 (conducted with 29 public health and 52 private pharmacies) and MAP 2015 (conducted with 80 public health facilities and 49 private pharmacies), a higher percentage of private pharmacies distribute short-acting contraceptives compared to public health facilities in Senegal." Within two years, pharmacies who reported selling contraceptives increased from $88 \%$ in 2013 to $100 \%$ in 2015 . This varied by the type of contraceptive method. In 2015, all the pharmacies surveyed sold oral contraceptives, including different brands. There was little difference between the proportion of pharmacies and public health facilities who reported selling injectable contraceptives ( $72 \%$ and $71 \%$ respectively). Among private pharmacies that did not sell injectable contraceptives, reasons included: no demand (50\%); method reserved for the public sector (29\%); product was never offered by distributors (14\%); and stopped the sale momentarily (7\%). Thirty-six percent of those pharmacies would be interested in selling injectables. In 2015, a higher proportion of private pharmacies surveyed (78\%) reported selling emergency contraceptives compared to public health facilities (63\%). Among private pharmacies who did not sell emergency contraceptives, 32\% would be interested in selling emergency contraceptives. Few private pharmacies reported selling implants and IUDs. 
Regarding the technical capacity of private pharmacies, in 2011, less than one fifth of the pharmacies' staff had "good" knowledge (staff could identify contraceptive methods and classify them as short or long acting, and by their effectiveness) of contraceptive methods and just over half had "average" knowledge (defined as the ability to cite the methods, but not being able to classify them). Half of the pharmacists and one-third of the counter agents had "good" knowledge of the classification of combined oral contraceptives. Approximately $50 \%$ of pharmacists and $75 \%$ of counter agents had a low level of knowledge of contraceptive side effects and situations requiring a referral. When a new client requested the pill, more than half of pharmacy staff sold the pill without a prescription.

\section{Conclusion}

A higher proportion of private pharmacies sell certain contraceptive methods compared to public health facilities. The technical capacity of private pharmacies' staff to offer FP services is insufficient and should be strengthened. Some private pharmacies offer FP methods illegally. Results from this analysis suggest that private pharmacy staff can play a larger role in providing FP services, beyond selling and giving guidance on drug indications, provided existing challenges are addressed. 


\section{Introduction}

Au Sénégal, l'indice synthétique de fécondité a eu tendance à se stabiliser autour de 5 enfants par femme entre l'Enquête Démographique et de Santé à Indicateurs Multiples (EDS-MICS) de 2005 et l'EDS continue (EDS-C) de 2014. Par contre, les taux de mortalité maternelle et infanto-juvénile ont considérablement diminué, passant respectivement de 401 à 392/100 000 naissances vivantes et de 121 à 54/1 000 pendant la même période.

Le taux de prévalence contraceptive moderne (TPCm), après avoir augmenté timidement jusqu'en 2010, a fait des bonds annuels de quatre (4) points de pourcentage à partir de 2012, pour atteindre 16\% en 2012 et 2013 et 20\% en 2014 (EDS-C 2014). Le rythme de progression a toutefois diminué en 2015 avec un TPCm rapporté de 21\% (EDS-C 2015). Malgré tout, les besoins non satisfaits en planification familiale (PF) restent encore importants. Par exemple, parmi les femmes en union, ils étaient estimés à 25\% en 2015 (EDS-C 2015).

Au Sénégal, notamment en milieu urbain, le nombre élevé, la proximité géographique et les horaires étendus des pharmacies ${ }^{1}$ en font souvent les premiers points de contact des populations avec le système de santé. Cependant leur potentialité d'offre de services de PF n'est pas assez exploitée. Selon l'EDS-C 2015, pour l'obtention des méthodes modernes de contraception, $84 \%$ des femmes sénégalaises s'adressaient au secteur public, mais un peu plus d'une femme sur dix, soit $11 \%$ avaient recours au secteur privé et le reste (seulement 1\%) s'orientait vers d'autres sources. Dans ce secteur privé, les pharmacies, qui représentent $9 \%$, sont de loin les principaux fournisseurs. Elles constituent ainsi, en milieu urbain, la deuxième source d'approvisionnement après les établissements du secteur public.

1 Au Sénégal, la dénomination dépend du secteur. Dans le secteur privé, en milieu urbain, les pharmacies sont dénommées " officines de pharmacie ", et plus communément appelées " officines ", ou "pharmacies privées ". En milieu rural, il y a les dépôts privés de médicaments. Dans le secteur public, l'appellation change suivant le niveau de la pyramide sanitaire : au niveau national, il y a la Pharmacie Nationale d'Approvisionnement (PNA) ; chaque région (11 régions/14) dispose d'une "Pharmacie Régionale d'Approvisionnement " (PRA); les districts sanitaires disposent d'un " Dépôt de district "; et au niveau des centres et postes de santé, on trouve un " dépôt " (de centre de santé, ou de poste). Les cases de santé sont desservies par le dépôt du poste de santé. Dans ce document " pharmacie " ou " pharmacie privée " réfère au secteur privé. 


\section{Contexte et justification}

En 2012, la Direction de la Santé de la Reproduction et de la Survie de l'Enfant (DSRSE) du Ministère de la Santé et de l'Action Sociale (MSAS) a adopté un ambitieux Plan d'action national de Planification Familiale (PANPF) pour la période 2012-2015. Ce PANPF visait à accroître le TPCm de $12 \%$ en 2012 à $27 \%$ en 2015, et $45 \%$ en 2020 . Cet objectif a été fixé conformément à l'engagement du pays d'atteindre les objectifs du millénaire pour le développement, en particulier les objectifs 4 et 5 . Le MSAS a renouvelé cet engagement à la déclaration FP2020 au Sommet de Londres en 2012. Le PANPF a adopté une approche multisectorielle faisant du secteur privé (à but lucratif et non lucratif) et du niveau communautaire, des partenaires clés du secteur public dans sa mise en œuvre. De ce fait, sur les 350000 nouvelles utilisatrices de PF prévues, 100000 devraient être recrutées par le secteur privé contre 125000 au niveau communautaire (PANPF, 2012).

Depuis 2012, le Programme PF a fait des progrès considérables grâce en partie à l'implication active du niveau communautaire et des organisations du secteur privé à but non lucratif dans la promotion et l'offre de services de PF. Cependant l'objectif des $27 \%$ de TPCm n'a pas été atteint en 2015, l'ESD-C rapportant un taux de 21\%. Cette situation est notamment due au fait que le secteur privé à but lucratif, y compris les pharmacies privées n'a pas été véritablement impliqué dans l'offre de services car la règlementation limite leur intervention en matière de PF à la vente des produits contraceptifs et à la fourniture de conseils $^{2}$ aux utilisateurs potentiels. Ainsi, les pharmacies privées sont exclues de l'offre effective des services de PF et ne peuvent qu'orienter les utilisateurs potentiels vers une structure publique de santé, un établissement du secteur privé à but non lucratif ou un médecin privé. Ceci est un obstacle à l'accès à la PF pour certaines personnes et certains couples. Pourtant, des expériences à travers le monde ont indiqué qu'au-delà d'être une source d'approvisionnement, les pharmacies privées peuvent jouer un rôle important dans l'offre de services. En plus, au niveau mondial, les évidences soutiennent un plus grand rôle des pharmacies et dépôts de médicaments dans l'offre des services du PF (USAID, 2013). Par exemple au Népal, l'EDS 2011 a montré que 12\% des femmes obtenaient leurs méthodes injectables dans les pharmacies, 52\% y achetaient des préservatifs et 32\% s'y approvisionnaient en pilules. De même, une étude de 2011 a montré qu'au Nigéria et en Ouganda, l'élargissement de la prestation de PF au secteur privé à but lucratif a permis de réduire les inégalités dans l'accès à la contraception moderne. En effet, l'EDS du Nigéria de 2013 , a montré que $38 \%$ des utilisatrices de la PF, achetaient leurs produits auprès des vendeurs de médicaments brevetés ${ }^{3}$ qui constituent la première source de PF.

Dans le cadre du Projet Evidence financé par I'USAID, le Population Council a entrepris en collaboration avec la DSRSE et I'USAID Sénégal, une étude intitulée "Exploration du rôle potentiel des pharmacies privées dans l'offre de services de PF au Sénégal ". Les évidences de cette étude pourront soutenir les efforts de plaidoyer en faveur d'une plus

2 Le "conseil " peut être défini ici comme une information donnée par rapportà un mal bénin ou une préoccupation particulière. Par contre le " counseling " est un élément clé des services de PF. II s'agit d'un mécanisme qui se fait en plusieurs étapes, et qui permet à la cliente d'obtenir de manière sûre et confidentielle toutes les informations relatives aux différentes méthodes de contraceptions disponibles afin de pouvoir faire un choix éclairé.

3 Au Nigéria, les vendeurs de médicaments brevetés sont des agents de santé à la retraite, comme des assistants en pharmacie, des infirmières, ou parfois des agents de santé communautaire qui vendent des médicaments après l'obtention d'une licence d'ouverture délivrée par le Conseil des pharmacies. 
grande implication des pharmacies privées dans l'offre de services de PF dans le cadre de l'approche multisectorielle adoptée par le Gouvernement du Sénégal afin de mieux répondre aux besoins de PF et de santé sexuelle et reproductive des personnes et des couples au Sénégal. Elle intègre plusieurs composantes, à savoir une revue documentaire des aspects juridiques (défis et opportunités) pour l'offre de services de PF par les pharmacies privées ; une revue documentaire des aspects non juridiques y compris une analyse secondaire des données disponibles sur les pharmacies privées ; une analyse secondaire des données issues d'études menées par l'Agence pour le développement du marketing social (ADEMAS), une organisation spécialisée en marketing social ; et une recherche qualitative auprès des parties prenantes clés de la PF et de pharmaciens privés de la région de Dakar. Chaque composante a fait l'objet d'un rapport spécifique (les trois autres composantes ainsi que la synthèse de l'étude sont disponibles sur le site du Projet Evidence). Le présent rapport porte sur la composante " analyse secondaire des données existantes de l'ADEMAS " sur les pharmacies privées afin d'obtenir une meilleure perception de leur expérience dans l'offre de services de PF. ADEMAS, un des partenaires de l'étude, qui travaille avec le secteur privé en utilisant des stratégies liées, entre autres, à l'accroissement de la demande et à l'accès aux produits et services de PF, mais aussi au renforcement de capacités en santé de la reproduction et PF du personnel des pharmacies (pharmacien, pharmacien-assistant et vendeur/agent de comptoir), a mené diverses études sur les pharmacies privées au Sénégal. Le présent document s'inscrit dans la perspective d'informer sur tous ces aspects. 


\section{Objectifs}

L'étude visait à fournir des évidences afin de soutenir le plaidoyer en faveur d'une plus grande implication des pharmacies privées dans l'offre de services de PF. L'analyse secondaire des données existantes de l'ADEMAS sur les pharmacies privées avait pour objectifs spécifiques de:

- Obtenir une meilleure perception de l'expérience des pharmacies privées en PF, à partir d'une analyse secondaire des données de l'ADEMAS sur les pharmacies privées

- Explorer les obstacles et opportunités des pharmacies privées à l'offre de services de $\mathrm{PF}$ en relation avec les variables disponibles dans les données de l'ADEMAS 


\section{Méthodologie}

L'activité a consisté en une analyse secondaire des données existantes de deux (2) études sur les pharmacies privées, notamment celles de l'enquête MAP (Mesure de l'Accès et de la Performance) de 2013 qui a été menée auprès de 29 formations sanitaires et 52 pharmacies privées dans les zones urbaines de Dakar, Thiès, Kaolack, Tambacounda, Saint-Louis, Diourbel et Ziguinchor, ainsi que celle de 2015 qui a été menée auprès de 80 formations sanitaires et 49 pharmacies privées dans les régions de Dakar, Thiès, Kaolack, Diourbel, Kaffrine et Kolda. Les bases de données des études susmentionnées ont été fournies et exploitées par ADEMAS pour une analyse secondaire. D'autres études ont également été exploitées, en l'occurrence, l'évaluation de l'effet de la formation par ADEMAS en technologie contraceptive des pharmaciens et agents des pharmacies privées du Sénégal sur la qualité de leurs prestations (2011).

II a été procédé à une analyse descriptive uni-variée et bi-variée des variables et une compréhension de la relation entre ces variables. 


\section{Résultats}

\section{Disponibilité des produits PF}

\section{Distribution des contraceptifs}

Dans les zones enquêtées (Dakar, Thiès, Kaolack, Diourbel, Kaffrine et Kolda) dans le cadre de la MAP 2015, la majorité (84\%) des points de vente éligibles constitués ici par les pharmacies privées et les formations sanitaires publiques (hôpitaux, postes et cases de santé), distribuent des produits PF. De manière générale, il apparaît que la contribution des pharmacies (100\%) dans la distribution de produits contraceptifs est plus importante que celle des formations sanitaires (74\%). Mais, quand on regarde les types de méthodes qui sont vendues dans les pharmacies et les formations sanitaires, on voit que certains produits sont plus disponibles dans les pharmacies et d'autres, dans les formations sanitaires. L'analyse des données laisse entrevoir, comme le montre le tableau ci-dessous, que les contraceptifs oraux (CO) sont présents dans la majorité, soit $83 \%$ des points de ventes concernés. Cependant, une analyse du type de canal de distribution, révèle que la proportion de pharmacies qui vend la $\mathrm{CO}$ est plus importante que celle des formations sanitaires. En effet, l'ensemble des pharmacies interrogées $(100 \%)$ distribuent des $C O$, là où près des deux tiers (73\%) des formations sanitaires le font. De manière globale, les injectables sont disponibles dans la majorité des points de vente visités. En effet, $72 \%$ déclarent vendre des injectables, mais, l'examen du type de canal de distribution, ne révèle que peu de différence dans les niveaux de distribution des pharmacies et des formations sanitaires qui sont sensiblement les mêmes, avec respectivement $72 \%$ et $71 \%$.

Globalement, les données de 2015 ont révélé une forte présence de la CU au niveau des points de vente concernés par l'étude dans la mesure où $68 \%$ d'entre eux déclarent en vendre.

\section{GRAPHIQUE 1}

DISTRIBUTION DES CONTRACEPTIFS EN 2015 AU NIVEAU DE DAKAR, THIÈS, KAOLACK, DIOURBEL, KAFFRINE ET KOLDA

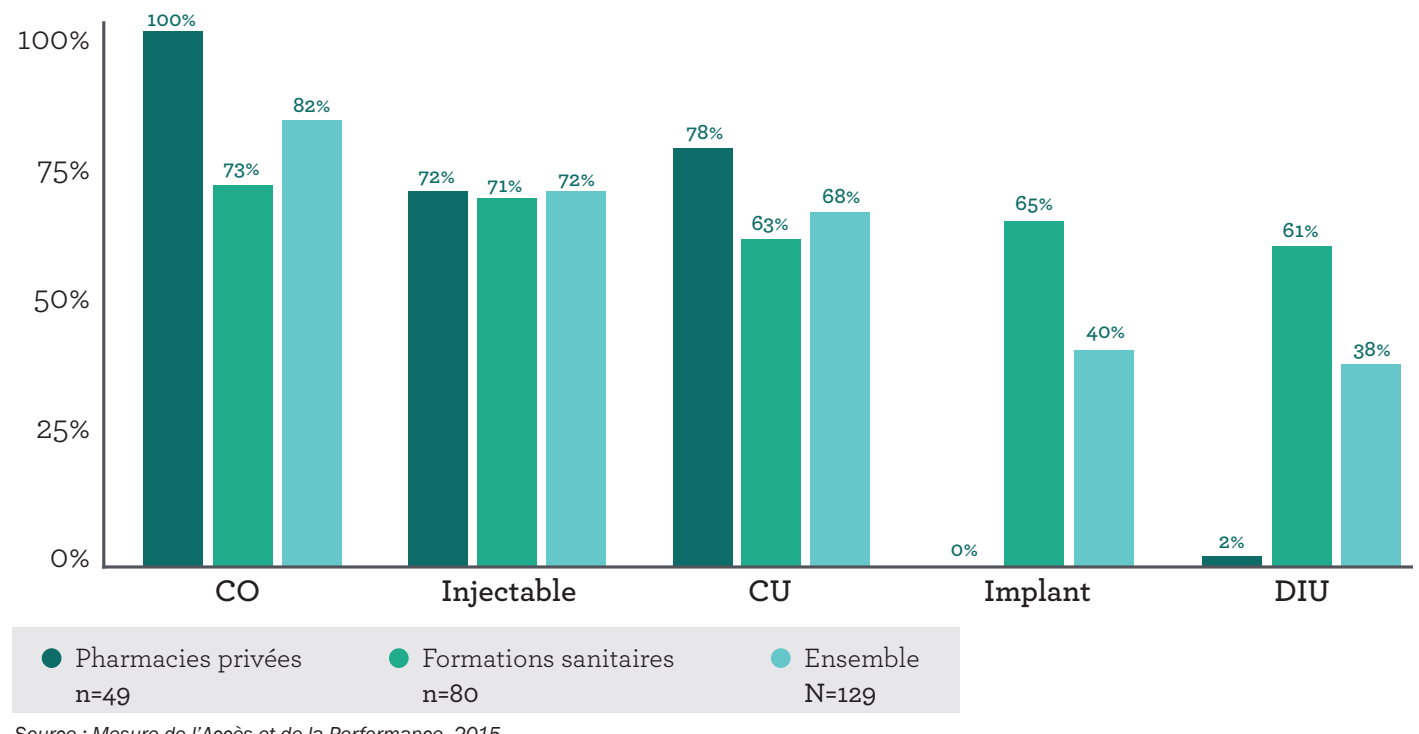

Source : Mesure de l'Accès et de la Performance, 2015 
En outre, si l'on considère le canal de distribution, on peut se rendre compte d'une présence plus marquée de la $\mathrm{CU}$ au niveau des pharmacies visitées (78\%) que dans les formations sanitaires (63\%). Parmi les structures visitées, $40 \%$ offrent les implants. Cependant, les pharmacies ne font pas partie de ces structures, alors que les $65 \%$ sont représentées par des formations sanitaires. En plus, selon les données, sur les 38\% des structures concernées par l'étude (pharmacies et formations sanitaires), très peu de pharmacies, distribuent le DIU. En effet, la distribution de ce produit reste dominée par les formations sanitaires avec $61 \%$ contre seulement $2 \%$ des pharmacies. Au niveau des pharmacies qui distribuent le DIU on retrouve essentiellement les trois (3) marques suivantes: PREGNA, PHARMATEXOVULE et OPTIMA.

\section{Les marques des contraceptifs distribuées dans les pharmacies}

Si l'on considère les marques de CO distribuées en pharmacie, il apparaît qu'elles sont très nombreuses. Hormis le Mycrogynon et le Luisa_F qui n'étaient pas disponibles au moment de l'enquête, toutes les autres marques habituellement distribuées au niveau des pharmacies étaient présentes même si c'était à des degrés divers. En effet, il est noté une forte présence du Sécuril qui est disponible au niveau de l'ensemble des pharmacies visitées, et dans une moindre mesure le Minidril (66\%) que l'on trouve dans un peu plus de la moitié des pharmacies. En dehors du Microlut qui est très peu distribué dans les pharmacies (2\%), d'autres marques (Stédéril, Trinoridiol, Microval, Adepal, Loféménal, et Jadel) y sont distribuées.

Comme pour les $\mathrm{CO}$, la vente d'injectables au niveau des pharmacies ne se limite pas à une seule marque. Les données font état d'au moins quatre (4) marques d'injectables disponibles au niveau de l'ensemble des pharmacies qui ont indiqué vendre cette méthode, notamment

\section{GRAPHIQUE 2}

MARQUES DE CONTRACEPTIFS ORAUX DISTRIBUÉES DANS LES PHARMACIES PRIVÉES (n=49) EN 2015

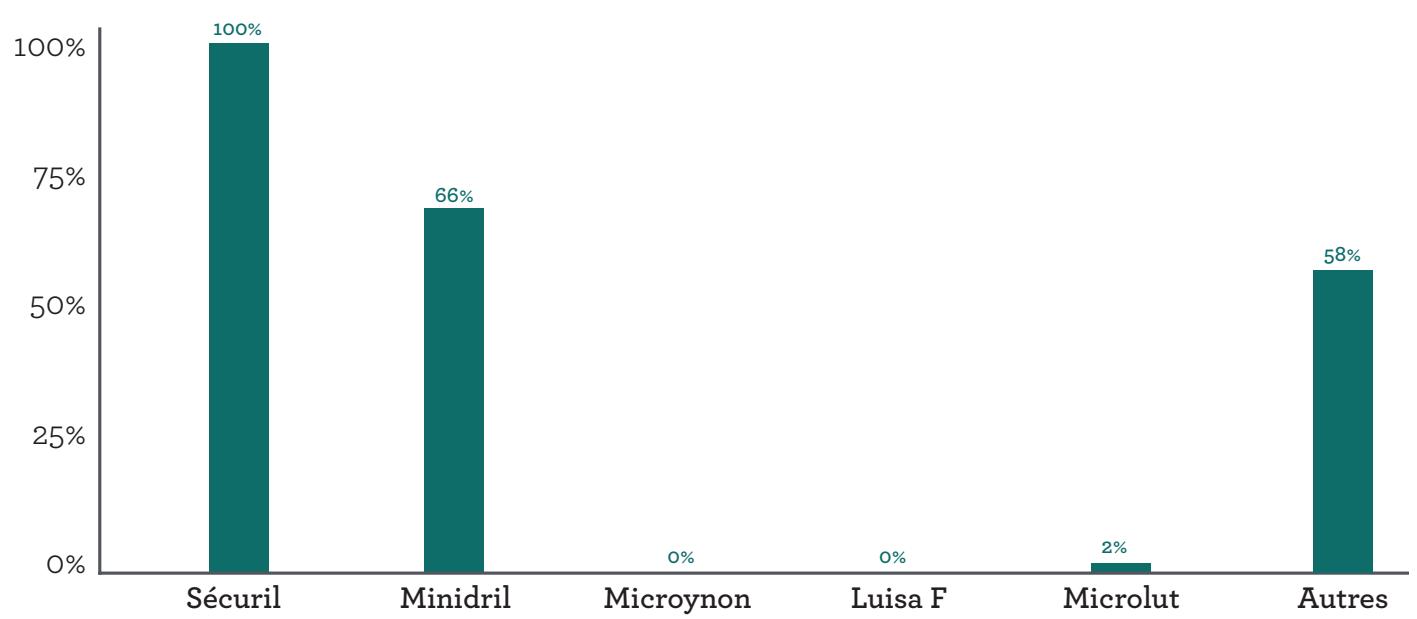


le Depo Provera et le Sécuril Press. D'autres marques telles que Norvel et Androtardin ont également été répertoriées dans toutes les pharmacies.

II ressort des résultats du Graphique 3 ci-dessous que de nombreuses marques de CU sont également distribuées en pharmacies. Même s'il faut reconnaître que le Norlevo constitue la marque la plus répandue puisque vendue par $78 \%$ des pharmacies, il n'en demeure pas moins que d'autres marques telles que Secufen et Linvo_BT ont été identifiées dans respectivement $10 \%$ et $8 \%$ des pharmacies. II en est de même pour le Norvel-72, Ellaone, et Today Pill qui représentent ensemble 36\% des autres marques distribuées au niveau des pharmacies.

\section{GRAPHIQUE 3}

MARQUES DE CONTRACEPTIFS D'URGENCE DISTRIBUÉES DANS LES PHARMACIES PRIVÉES ( $\mathrm{n}=49$ )

\section{EN 2015}

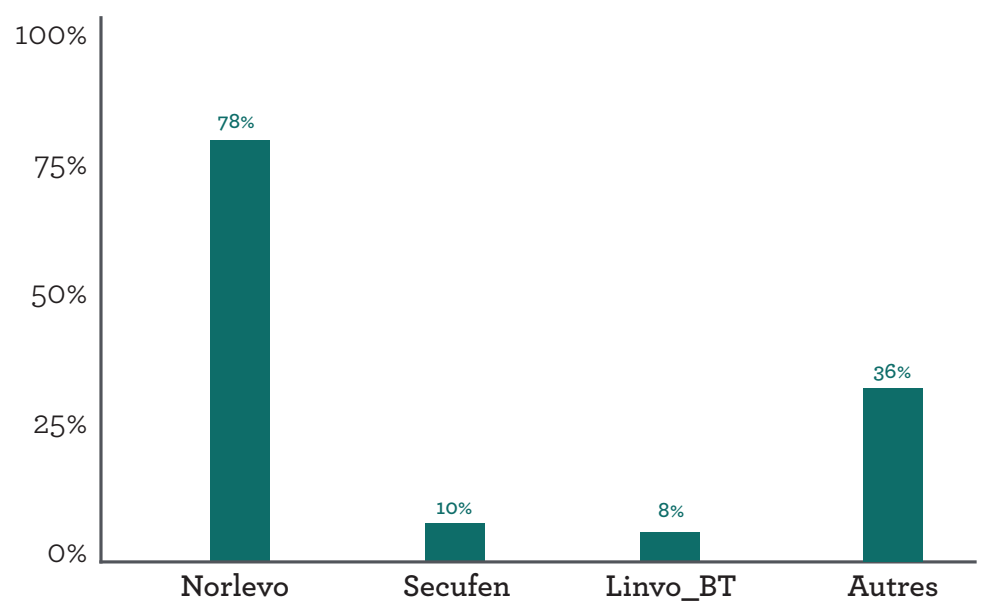

Source : Mesure de l'Accès et de la Performance, 2015

Au niveau des pharmacies qui distribuent le DIU, on retrouve essentiellement les trois (3) marques suivantes : PREGNA, PHARMATEXOVULE et OPTIMA. Aucune pharmacie privée visitée ne vendait d'implants.

\section{Principales raisons de la non-vente des contraceptifs dans les pharmacies privées}

Les $28 \%$ de pharmacies qui affirment ne pas vendre de contraceptifs injectables ont avancé plusieurs raisons (Graphique 4). La principale raison évoquée par la moitié d'entre eux (50\%) est l'absence de demande des populations pour cette méthode. Pour d'autres (29\%), il s'agirait d'une méthode réservée au secteur public de la santé, tandis que 14\% d'entre eux déclarent que le produit ne leur avait jamais été proposé par les distributeurs. Une faible proportion, mais non moins négligeable (7\%) affirme avoir arrêté momentanément de vendre la méthode sans donner explicitement les raisons de cet arrêt. 
L'examen des raisons évoquées dans les différentes régions, fait apparaître des disparités. En effet, c'est seulement dans la région de Dakar, que toutes les raisons susmentionnées ont été avancées. L'absence de demande est la raison commune à toutes les régions, à l'exception de Thiès où les pharmaciens déclarent ne pas vendre cette méthode à leur niveau, parce qu'ils considèrent qu'il s'agit d'un produit réservé au seul secteur public. L'absence de demande constitue également la seule raison avancée dans les régions de Kolda, Diourbel et Kaolack. Par ailleurs, en dehors de Dakar, c'est dans la région de Kaffrine que les pharmaciens ont affirmé n'avoir jamais reçu de proposition pour vendre les injectables.

Pour $22 \%$ des pharmacies qui déclarent ne pas vendre la $\mathrm{CU}$, trois (3) principales raisons ont été évoquées pour justifier cette attitude. Si la majorité des pharmacies (55\%) ont décidé de ne pas vendre la CU, c'est tout simplement parce qu'ils n'ont jamais reçu de proposition de la part des distributeurs pour cette méthode. Par contre, pour d'autres (36\%), c'est parce qu'ils n'ont pas assez de demande, tandis que pour quelques-uns (9\%), cette méthode est réservée au secteur public (Graphique 4).

Comme pour les autres méthodes, plusieurs raisons ont été évoquées au niveau des pharmacies, pour expliquer la non-distribution des implants à leur niveau. Si pour la majorité (48\%), il n'y a pas de demande pour cette méthode, certains (30\%) affirment n'avoir jamais reçu de proposition dans ce sens pendant que d'autres (20\%) seraient d'avis que cette méthode est réservée au secteur public de la santé. Concernant les pharmacies qui ne distribuent pas le DIU, la raison évoquée par la plupart d'entre elles (41\%) est l'absence de demande suffisante. Par contre pour un peu plus du quart des personnes interrogées (29\%), c'est parce qu'elles considèrent qu'il s'agit d'un produit réservé au secteur public de la santé et à peu près la même proportion (26\%) affirme n'avoir jamais reçu de proposition de vente pour ce produit.

\section{GRAPHIQUE 4}

RAISONS DE LA NON-VENTE DES CONTRACEPTIFS DANS LES PHARMACIES PRIVÉES ( $\mathrm{n}=49$ ) PAR MÉTHODE EN 2015

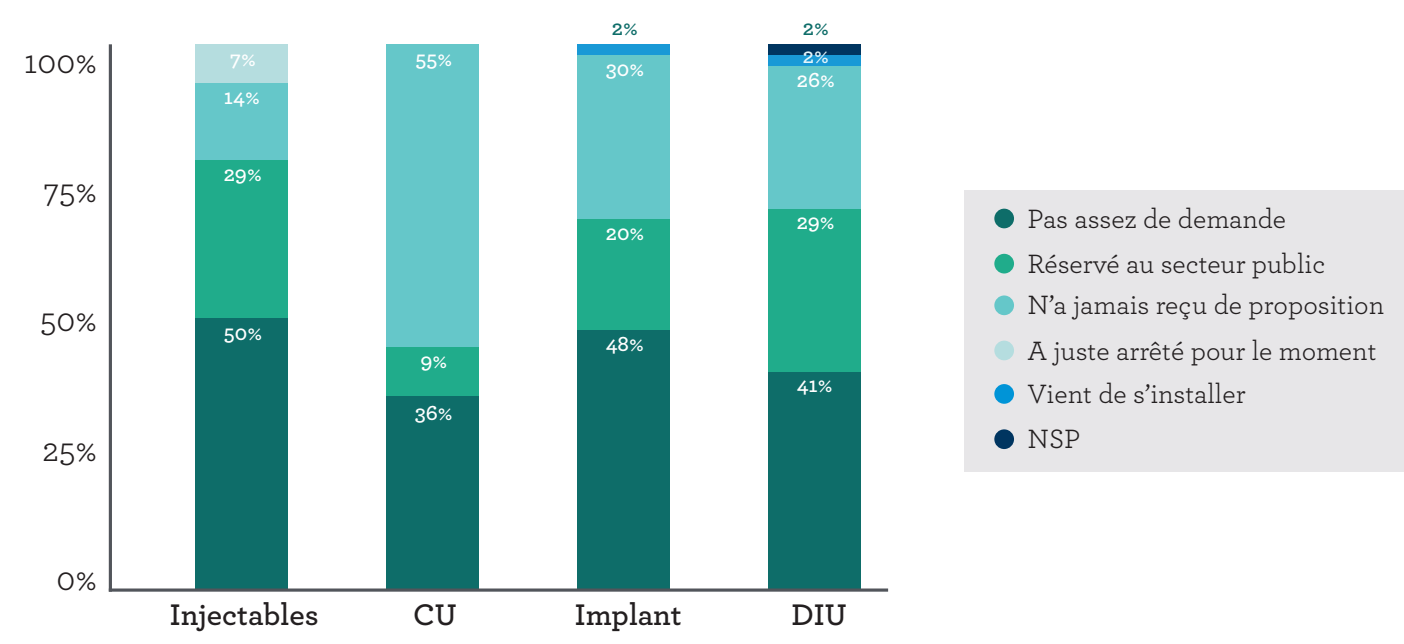

Source : Mesure de l'Accès et de la Performance, 2015 


\section{Potentiel de vente de contraceptifs au niveau des pharmacies privées}

Àla question de savoir si les pharmacies qui ne vendent pas d'injectables, seraient intéressées par la vente de ce produit, l'analyse des données montre que même si 50\% d'entre elles ont répondu par la négative, $14 \%$ ne se sont pas prononcés sur la question. Concernant la question de savoir si les pharmacies qui ne vendent pas de CU, seraient intéressées par la vente de ce produit, le Graphique 5 ci-dessous montre que l'intérêt suscité par cette idée, n'est pas très manifeste puisque seuls 32\% répondent par l'affirmative contre $53 \%$ qui ne sont pas intéressées par ce produit.

\section{GRAPHIQUE 5}

POTENTIEL DE VENTE DES CONTRACEPTIFS DANS LES PHARMACIES PRIVÉES (n=49) EN 2015

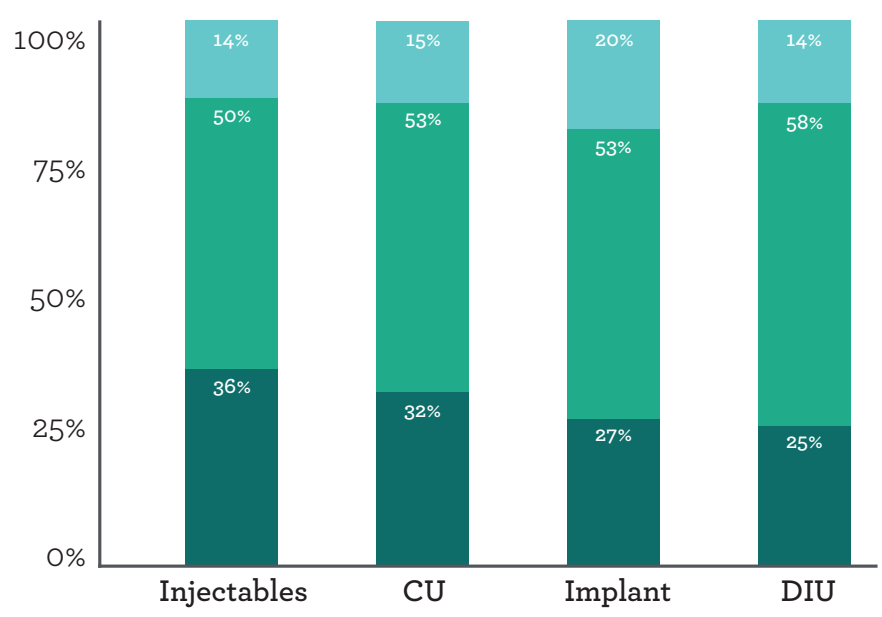

Intéressé par la vente

- Non intéressé

NSP

Source : Mesure de l'Accès et de la Performance, 2015

II ressort du graphique ci-dessus qu'un peu plus de la moitié (53\%) des pharmacies ne sont pas intéressées par la vente d'implants. Toutefois, même si une faible proportion d'entre eux (27\%) se dit intéressée par la vente de ce produit, il faut tout de même noter qu'une partie non négligeable (20\%) ne s'est pas prononcée sur la question. Concernant la question de savoir si les pharmacies qui ne vendent pas de DIU, seraient intéressées par la vente de ce produit, l'analyse des données montre qu'un peu plus de la moitié (58\%) ont répondu par la négative.

\section{Évolution de la distribution de contraceptifs dans les pharmacies privées entre 2013 et 2015}

Au regard des données disponibles, il apparaît que les pharmacies se sont de plus en plus intéressées à la distribution de produits contraceptifs. En effet, l'offre de produits contraceptifs au niveau de ces structures a beaucoup évolué puisqu'en l'espace de deux (2) ans, le nombre de pharmacies impliquées dans la distribution de produits contraceptifs a nettement progressé. Alors qu'il ne représentait que 88\% en 2013 il est passé à 100\% 
en 2015, soit une évolution de 12 points. On note une nette évolution du pourcentage de pharmacies privées qui vendent des $\mathrm{CO}$. En effet, la proportion s'est beaucoup améliorée passant de $85 \%$ en 2013 à la totalité (100\%) en 2015. En revanche, pour les injectables, il apparaît une légère baisse dans l'offre de ce produit au niveau des pharmacies qui est passée de $75 \%$ en 2013 à $72 \%$ en 2015, soit une chute de 3 points durant cette période.

\section{Analyse des parts de marché des contraceptifs ( $\mathrm{CO}$ et injectables)}

Les parts de marché sont traduites ici en "volume " et en "valeur ". Le volume du marché représente les estimations des volumes annuels de vente de chaque canal (pharmacies privées et formations sanitaires). Ainsi, au niveau de chaque canal, les estimations sont faites d'abord par point de vente avant d'agréger l'ensemble des points de vente pour estimer le volume de vente du canal. La valeur du marché, quant à elle, représente les estimations annuelles de la valeur marchande des produits vendus ou distribués par canal. Ces estimations sont basées sur les prix appliqués aux différents produits vendus. Les résultats de la MAP 2015 montrent des parts de marché de $\mathrm{CO}$ en volume en pharmacie de l'ordre de $9 \%$ et $53 \%$ de la valeur de chaque canal. Les injectables représentent $3 \%$ du volume vendu en pharmacie et $18 \%$ de la valeur. II en ressort que le secteur public a une plus grande part de marché de l'injectable que les CO. Ceci s'entend dans la mesure où l'injectable nécessite l'accompagnement d'un prestataire de santé alors que les CO peuvent être consommés sans la présence systématique d'un prestataire.

\section{Capacités techniques du personnel des pharmacies privées à offrir des services PF}

Entre 2008 et 2011, l'ADEMAS a organisé 28 sessions de formations dans 11 régions du pays dans le cadre du programme 2006-2011 de I'USAID. Ces formations avaient pour objectif de renforcer les capacités du personnel des pharmacies privées en technologies contraceptives et infections sexuellement transmissibles/syndrome de l'immunodéficience acquise (IST/SIDA). Elles ont permis de former 137 pharmaciens et 829 vendeurs/agents de comptoirs sur :

- Les différentes méthodes de contraception (mode d'action et efficacité);

- La CO (types de pilules combinées et équivalences, avantages et inconvénients, mode d'utilisation, suivi et conseils pour le réapprovisionnement) ;

- La CU (méthodes et conseils utiles) ; et,

- La communication interpersonnelle (composantes, causeries éducatives et counseling en PF).

L'évaluation de ces formations qui avait pour objectif général de mettre en évidence la contribution des pharmacies privées dans l'offre de service de PF a permis de mettre en exergue les constatations suivantes. 


\section{Niveau de connaissance des méthodes PF par le personnel des pharmacies privées}

De façon générale, le Graphique 6 ci-dessous montre qu'en moyenne, moins d'un cinquième du personnel des pharmacies avait un bon niveau de connaissance (a pu faire une bonne classification) des méthodes et qu'un peu plus de la moitié avait une connaissance plutôt moyenne (le personnel a pu les citer les méthodes sans pouvoir les classer). Ces résultats sont similaires à ceux obtenus au niveau des différentes régions visitées.

GRAPHIQUE 6

NIVEAU DE CONNAISSANCE DES MÉTHODES PF PAR LE PERSONNEL DES PHARMACIES PRIVÉES EN 2011

Pharmacien

Très Bon

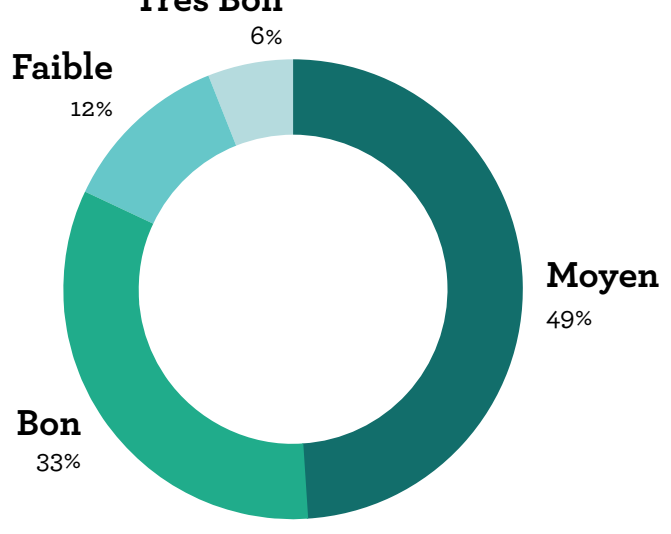

Agent de comptoir

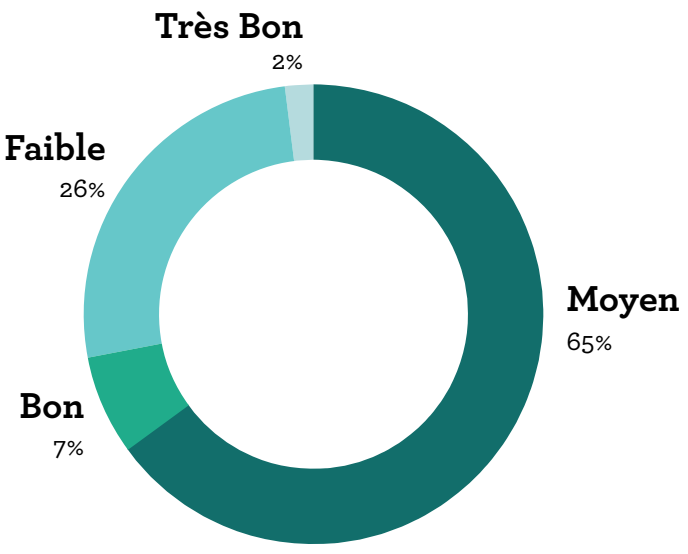

Source : Évaluation de la formation par ADEMAS en technologies contraceptives et infections sexuellement transmissibles/syndrome de l'immunodéficience acquise (IST/SIDA) des pharmaciens et agents des officines privées du Sénégal sur la qualité de leurs prestations, 2012

\section{Niveau de connaissances sur la classification des types de pilules combinées}

Les données révèlent que la moitié des pharmaciens (50\%) avait une bonne connaissance de la classification des différents types de pilules combinées et que les autres (50\%) en avait une connaissance moyenne. Par ailleurs, on constate que seul un tiers des agents de comptoirs avait une bonne connaissance de cette classification, et que 70\% d'entre eux en avaient une connaissance moyenne. Ainsi, les pharmaciens maîtrisent mieux la classification des types de pilules combinées parce qu'ils se préoccupent de la qualité du produit et ses attributs alors que les agents de comptoirs ne sont focalisés que sur la fonction vente du produit.

Au niveau des régions, on remarque que les proportions de personnel de pharmacies ayant une bonne connaissance de la classification des pilules combinées étaient pour la plupart faibles, allant de $12 \%$ à 41\%, sauf à Tambacounda où une part importante du personnel des pharmacies (71\%) avait une bonne connaissance de cette classification. La proportion la plus faible a été enregistrée dans la zone de Fatick- Diourbel (12\%). 


\section{GRAPHIQUE 7}

PROPORTIONS DE PERSONNEL DE PHARMACIES (N=102) AYANT LA BONNE CONNAISSANCE DE LA

CLASSIFICATION DES TYPES DE PILULES COMBINÉES PAR RÉGION

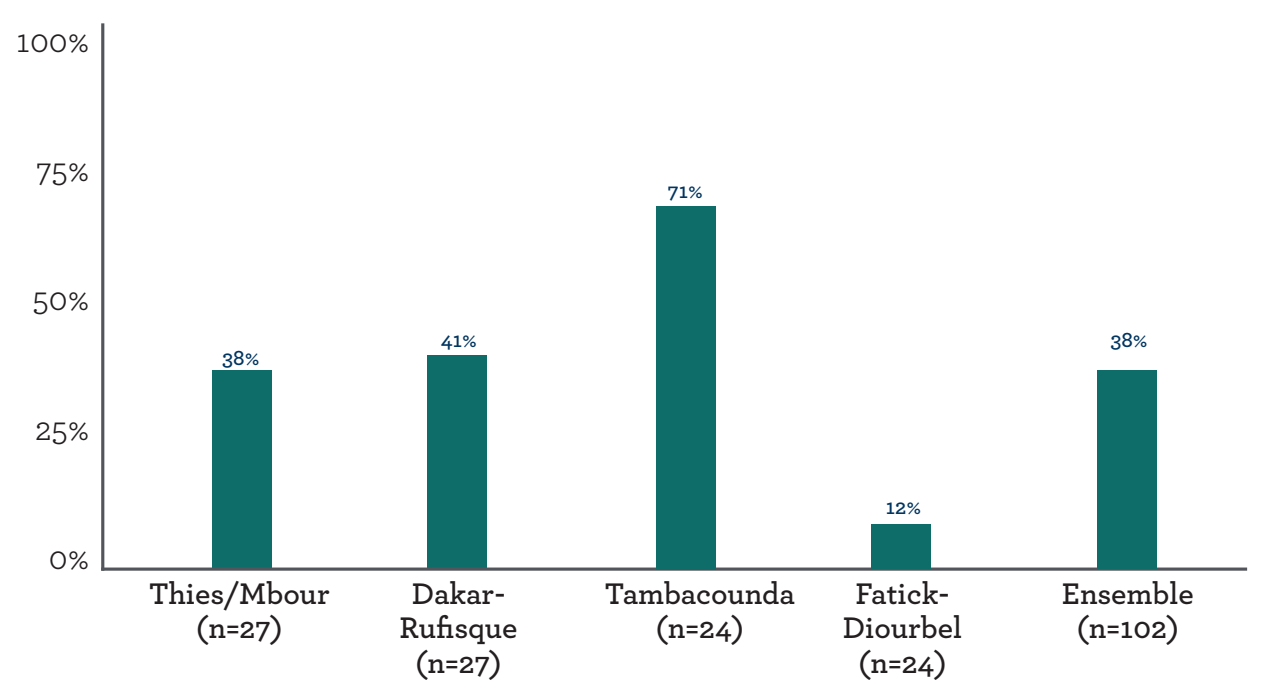

Source : Mesure de l'Accès et de la Performance, 2015

\section{Niveau de connaissance des effets secondaires ou situations nécessitant une référence}

Les données du Graphique 8 ont montré que le personnel des pharmacies n'avait pas une bonne maîtrise des effets secondaires des produits contraceptifs. En effet, très peu parmi ces agents, avaient une bonne connaissance des effets secondaires ou des situations nécessitant une référence. Alors que la moitié (50\%) des pharmaciens avaient une faible connaissance des effets secondaires ou situations nécessitant une référence, près de huit (8) agents de comptoirs sur $10(76 \%)$ avaient une connaissance très faible (voire, presque nulle) de ces effets secondaires ou situations.

\section{Attitude du personnel face à une demande de CO}

Face à une demande de pilule pour une première utilisation de la PF, plus de la moitié du personnel des pharmacies 55\% n'avait pas la bonne attitude car ne respectait pas la législation en vigueur. En effet, ce personnel est allé au-delà de ses attributions en délivrant la pilule sans présentation d'une ordonnance venant d'un prescripteur agréé. L'analyse révèle par contre qu'en cas de réapprovisionnement, 92\% du personnel des pharmacies adoptaient une bonne attitude en faisant de la présentation de l'ordonnance une exigence pour l'achat du produit. 
GRAPHIQUE 8

NIVEAU DE CONNAISSANCE DES EFFETS SECONDAIRES OU SITUATIONS NÉCESSITANT UNE RÉFÉRENCE EN 2011

\section{Pharmacien}

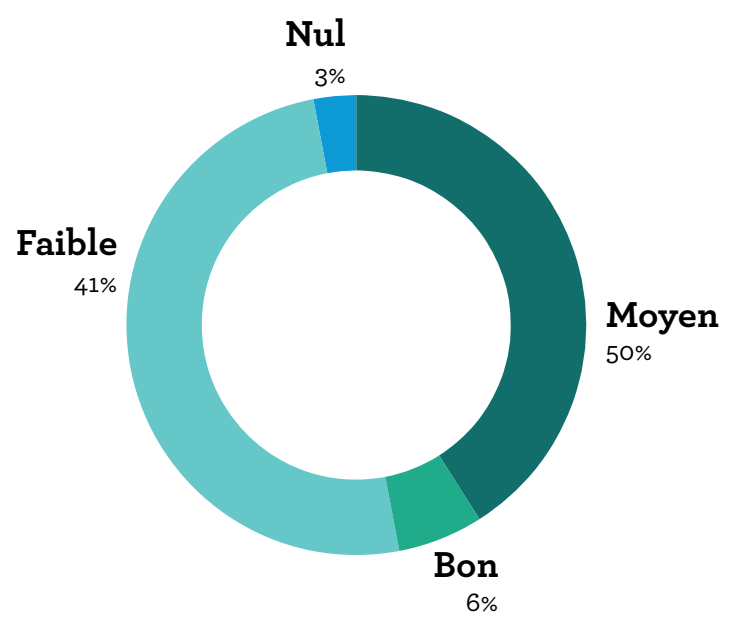

Agent de comptoir

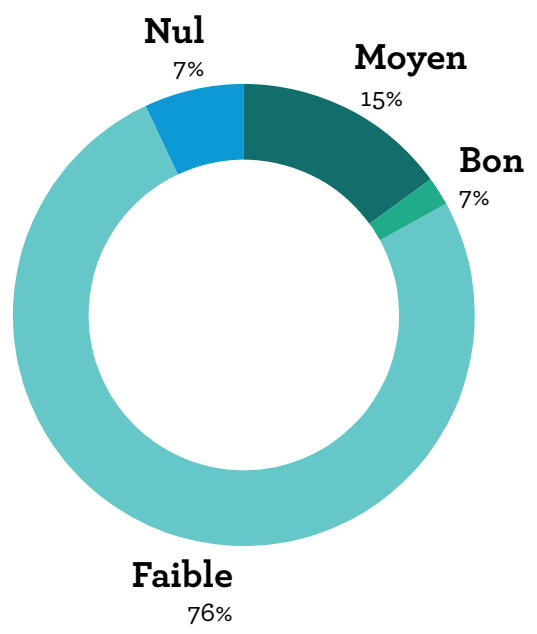

Source : Évaluation de la formation par ADEMAS en technologies contraceptives et IST/SIDA des pharmaciens et agents des officines privées du Sénégal sur la qualité de leurs prestations, 2012 


\section{Discussion}

Lorsqu'on examine les différents canaux de distribution, il apparaît que la contribution en termes de vente de produits de PF du secteur privé, notamment les pharmacies, est très considérable dans la mesure où elle est plus importante que celle notée au niveau du secteur public. Et cela s'est fait progressivement, durant ces dernières années dans les régions visitées, le nombre de pharmacies impliquées dans la distribution de produits contraceptifs ayant fortement augmenté jusqu'à atteindre le maximum pour certaines méthodes (particulièrement les $\mathrm{CO}$ ).

Même si toutes les méthodes disponibles au niveau du programme PF ne le sont pas au niveau des pharmacies, on peut toutefois y retrouver les plus connues, à savoir les $\mathrm{CO}$, avec notamment une forte présence de la CU, des injectables, et du DIU. Comparée aux structures de santé publique, on peut noter une importance numérique de certaines méthodes au niveau des pharmacies, mais aussi l'existence de nombreuses marques. II faut tout de même reconnaître qu'en fonction des différentes méthodes, cette disponibilité peut s'observer à des degrés divers. En effet, les $\mathrm{CO}$ sont les plus répandus puisque l'ensemble des pharmacies en vendent. Les injectables sont également bien distribuées, mais dans une moindre mesure. Par contre, peu de pharmacies distribuent le DIU, tandis que les implants n'ont été retrouvés dans aucune d'entre elles. Concernant les pharmacies qui ne distribuent pas ces méthodes, les raisons sont essentiellement liées au fonctionnement du marché, à savoir l'absence de demande pour ces méthodes ou le fait que le produit n'ait jamais été proposé par les distributeurs, mais aussi à l'existence de certains préjugés puisque certains pharmaciens pensent qu'il s'agit de méthodes réservées au secteur public de la santé. Cette situation explique sans doute l'intérêt mitigé pour la vente de ces méthodes, même s'il faut noter qu'au niveau des pharmacies, une proportion non négligeable de personnes interrogées n'a pas souhaité se prononcer sur le potentiel de vente de ces méthodes.

Concernant les capacités du personnel des pharmacies à offrir les services de PF, l'analyse a permis de relever des insuffisances dans les connaissances des différentes méthodes malgré la formation reçue, mais aussi des effets secondaires, alors même qu'il est très attendu dans le rôle de conseils utiles, voire de référence chaque fois que le besoin se ferait sentir. Cependant, leurs attitudes pratiques face à un besoin de PF, montrent que malgré tout, une part importante de pharmacies privées offre, de manière non-officielle, certaines méthodes de PF en dépit des restrictions juridiques. 


\section{Limites de l'étude}

Avec les études MAP, le type d'analyse fondé sur la distribution par région et le milieu de résidence n'était pas assez pertinent, et n'a pas toujours été possible en ce sens que le nombre de pharmacies interrogées dans le cadre des études MAP était très inégalitaire selon les régions. Par conséquent, une comparaison des niveaux de distribution n'aurait pas été très correcte. Par ailleurs, les pharmacies se trouvent le plus souvent en milieu urbain, contrairement au milieu rural où l'on trouve des dépôts pharmaceutiques. Concernant le renforcement des capacités des pharmaciens en matière de PF, seules quatre (4) zones sur les 11 régions concernées par la formation ont été choisies pour l'évaluation. 


\section{Recommandations}

\section{À l'endroit du MSAS/DSRSE}

- Soutenir le plaidoyer pour la mise en place d'un cadre juridique favorable en collaboration avec l'Ordre et le Syndicat des Pharmaciens afin de mieux impliquer le personnel des pharmacies dans l'offre initiale de services de PF.

- Organiser pour les pharmaciens des formations appropriées en technologie contraceptive conformément aux politiques, normes et protocoles.

\section{$\grave{A}$ l'endroit de l'Ordre et du Syndicat des Pharmaciens}

- Soutenir le plaidoyer pour la mise en place d'un cadre juridique favorable à l'offre initiale de méthodes de PF par les pharmacies privées.

\section{À l'endroit des pharmaciens}

- Aménager au sein des pharmacies un espace adéquat pour le counseling et l'administration de méthodes de PF. 


\section{Conclusion}

L'analyse secondaire des données tirées des études de l'ADEMAS a permis d'avoir un meilleur aperçu de la disponibilité des produits, du volume et de la qualité des interventions des pharmacies privées en matière de PF, mais également de leurs capacités, perceptions et attitudes. II est évident qu'en dépit des obstacles, il existe de réelles possibilités d'accroître leur contribution dans l'offre de services de PF. Avec une taille de marché potentielle estimée en 2014 à plus de 1,1 million de femmes qui ont besoin de PF au Sénégal, les pharmacies privées constituent d'excellents points de contact pouvant servir à informer, et mieux, offrir des services de PF aux populations. 


\section{Références bibliographiques}

ADEMAS. 2013. Enquêtes MAP (Mesure de l'Accès et de la Performance).

ADEMAS. 2015. Enquêtes MAP (Mesure de l'Accès et de la Performance).

ADEMAS. 2016. Analyse de la situation en rapport avec l'Approche du Marché Total des produits contraceptifs.

Agence Nationale de la Statistique et de la Démographie (ANSD) [Sénégal], et ICF International. 2015. Sénégal : Enquête Démographique et de Santé Continue (EDS-Continue 2014). Rockville, Maryland, USA : ANSD et ICF International.

Agence Nationale de la Statistique et de la Démographie (ANSD) [Sénégal], et ICF International. 2016. Sénégal : Enquête Démographique et de Santé Continue (EDS-Continue 2015). Rockville, Maryland, USA : ANSD et ICF International.

Dieng A. 2012. Evaluation de la formation par ADEMAS en technologies contraceptives et IST/SIDA des pharmaciens et agents des officines privées du Sénégal sur la qualité de leurs prestations. Mémoire de fin de formation pour l'obtention du Diplôme d'Etudes Supérieures Spécialisées en Gestion des Services de Santé, option Gestion des Programmes de Santé.

Plan d'Action National de Planification Familiale 2012-2015. République du Sénégal. Dakar : MSAS.

Enquêtes MAP (Mesure de l'Accès et de la Performance) de 2013 et de 2015. 


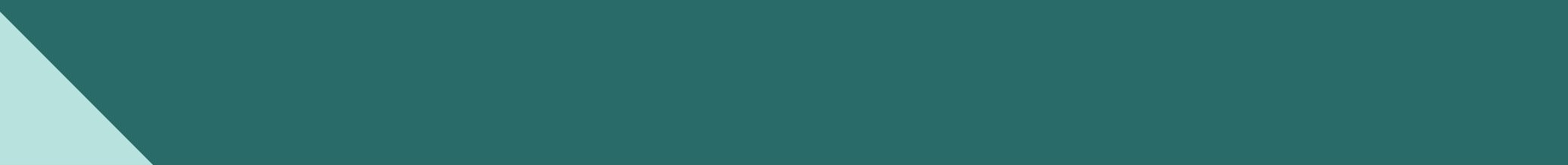

